\title{
ANALYSING THE GROWTH CONDITION OF HAZEL MUSHROOM BASE ON THE DATE OF LANDSAT 8 AND THE PLATFORM OF ENVI
}

\author{
Juntao Zhu ${ }^{1}$, Yang Luo ${ }^{1}$,Miaoxing Zhao ${ }^{1}$, Lei Wang ${ }^{1}$, Chaofei Gong ${ }^{1}$, Yusheng Liu \\ Guilin University of Technology, College of Geomatics and Geoinformation, 541006, Guilin
}

KEY WORDS: Armillariella mellea, Remote Sensing, Artificial cultivation, Atmospheric Correction, LST, TVDI

\begin{abstract}
:
Armillariella mellea mainly distributes in Changbai Mountain forest area, which is one of the few edible fungi that can be cultured artificially. It contains a variety of essential amino acids and vitamins for human body. Frequent consumption can strengthen the body immunity. It is of great significance to analyze the growth environment of Armillariella mellea by remote sensing technology for its growth prediction and artificial cultivation research. Based on ENVI software and Landsat 8 image, the surface temperature and soil moisture in Xiao Hinggan Mountains in August 2014 were retrieved by the method of atmospheric correction and TVDI, and the optimum growth environment of Armillariella mellea was analyzed. The results are as follows: 1) The optimum growth temperature of Armillariella mellea is $25-30^{\circ} \mathrm{C}$, and the soil moisture condition is $0.4-0.6$. The Armillariella mellea mainly distributes in the north-central part of the study area. Combined with other growth environment information, the study area is generally suitable for the growth of Armillariella mellea; 2) we found the Armillariella mellea around the area of 83 samples of 100 samples which were choose to analyse. The accuracy is higher; 3) It is feasible to obtain the optimum growth environment of Hazelnut mushroom by remote sensing technology.
\end{abstract}

\section{INTRODYTION}

Hazelnut mushroom is the fruiting body of Armillaria mellea. It mainly grows in Heilongjiang, Jilin, Liaoning and Inner Mongolia provinces and regions(Zheng et al., 2014). It is a famous edible and medicinal fungus, and is a kind of precious and natural health food. Due to the influence of natural factors, its annual growth will be different. The analysis of the growth environment of Hazelnut mushroom by remote sensing technology is conducive to the artificial cultivation of Hazelnut mushroom in the future.

There have been many studies on calculating surface temperature and soil moisture using high resolution remote sensing images at home and abroad, and different models and methods have been put forward. The traditional method of surface temperature measurement using thermometers can only measure local temperature, but also has some limitations, which wastes time and manpower [Zhu et al., 2016]. At present, many scholars have studied the theory and application of Land surface temperature (LST) inversion based on thermal infrared band, and proposed a series of LST inversion algorithms [Zhu et al., 2006].The representative LST inversion algorithms are: single channel algorithm [Jimenez-Munoz et al., 2009], single window algorithm [Qin et al., 2001], split window algorithm [Rozenstein et al., 2014]. Soil moisture is an important parameter in the field of agriculture and forestry. Researchers have done a lot of research on remote sensing monitoring of soil moisture, and put forward different models and methods, including apparent thermal inertia method [Yu et al., 2011], temperature value by drought index [Holzman et al., 2014], vegetation water supply index [Zhou et al., 2012], microwave polarization difference index [Gao et al., 2017], Integral equation model [An et al., 2017], Dobson model [Wang et al., 2017]. Microwave remote sensing is only suitable for smallscale soil moisture inversion [Yan et al., 2006]. Compared with traditional soil moisture monitoring methods, remote sensing technology can quickly acquire soil moisture status in a large area, which is of great significance for agricultural and forestry drought monitoring. In summary, the technology of extracting surface temperature and soil moisture from remote sensing images is relatively mature. How to combine the two technologies has certain research significance for obtaining hazelnut mushroom information. Taking winter wheat as an example, using remote sensing technology to monitor crop growth can provide timely information for field management and estimate yield [Wu et al., 2004]. Based on the division of crop planting structure by using remote sensing technology and the method of combining cluster sampling and spline sampling, the crop planting area can be estimated [Yang et al., 2007]. The analysis of biological characteristics, nutrient components and pharmacological effects of Armillaria mellea can meet the needs of hazelnut mushroom market at home and abroad, and provide a basis for further study of Armillaria mellea [Zhenget al., 2007]. Therefore, it is feasible to extract the information of Hazelnut mushroom growth environment by using remote sensing technology. In this paper, Landsat 8 image is used to retrieve surface temperature and soil moisture in ENVI, and determine its threshold range. Then the growth area of Hazelnut mushroom is effectively obtained by combining other growth information. Finally, the results are analyzed and evaluated to provide scientific data for artificial breeding and growth prediction of Hazelnut mushroom.

\section{DATA RESOURCES AND STUDY AREA}

\subsection{Date Resources}

Landsat is a series of satellites launched and managed jointly by NASA and USGS. Landsat has been widely used in detecting earth resources, investigating underground mineral deposits, marine resources and groundwater resources, monitoring and managing agriculture, forestry, animal husbandry and water resources in the United States. There are a lot of achievements in predicting crop yields, studying the growth and landform of natural plants, investigating and forecasting various serious natural disasters and environmental pollution. Landsat- 8 satellite is the eighth satellite of Landsat series satellites, 
carrying OLI land imager and TIRS thermal infrared sensor, which was successfully launched by NASA on February 11, 2013. Compared with ETM on Landat-7, OLI made adjustments in Band5 band to exclude the effect of water vapor absorption at $0.825 \mu \mathrm{m}$; shortened the Band 8 band to distinguish vegetation from non-vegetation areas; and increased Band 1 blue band and Band 9 short-wave infrared band for coastal zone observation and cloud detection. The TIRS thermal infrared sensor carried by LandSat- 8 is mainly used to collect heat loss in two hot zones of the earth to understand the water consumption in the observed zones. In this paper, Landsat 8 image of Xiao Hinggan Mountains on August 25, 2014 was acquired by using geospatial data cloud website. The cloud coverage rate was $2.48 \%$. Its quality was good. The distribution of hazelnut mushroom in the study area was extracted based on ENVI platform, and the results were analyzed and evaluated.

\subsection{Study Area}

The study area is located in the north of Heilongjiang Province, China. It covers 48.3-49.1 degrees north latitude and 128.2129.2 degrees east longitude(Figure 1). Its terrain shows a northwest-southeast trend. The northwest is connected with the Yile Huli Mountains, a branch of the Great Hinggan Mountains, and the southeast is to the north end of Zhangguangcai Mountains along the Songhua River. It is about $450 \mathrm{~km}$ long in North and south, $210 \mathrm{~km}$ wide in East and west, and has an area of $77,725 \mathrm{~km} \mathrm{2}$. Its geological structure is complex, and its altitude is generally between 500 and 800 . It belongs to the type of low hills and mountains, with gentle slopes and wide valleys. The annual average temperature is $-2 \sim 2 \mathrm{C}$, the annual average rainfall is $500 \mathrm{~mm}$, and the frost-free period is about 100 days. Many precious trees, such as Korean pine, grow and multiply, and become the national key timber forest base. The stock of Korean pine is about 450 million cubic meters. At present, the stock of Korean pine is more than 430 million cubic meters, which accounts for more than half of the total stock of Korean pine in China. It is known as the "hometown of Korean pine". In the dense forest, there are many rare birds and animals, wild plants and fungi are rich in resources.

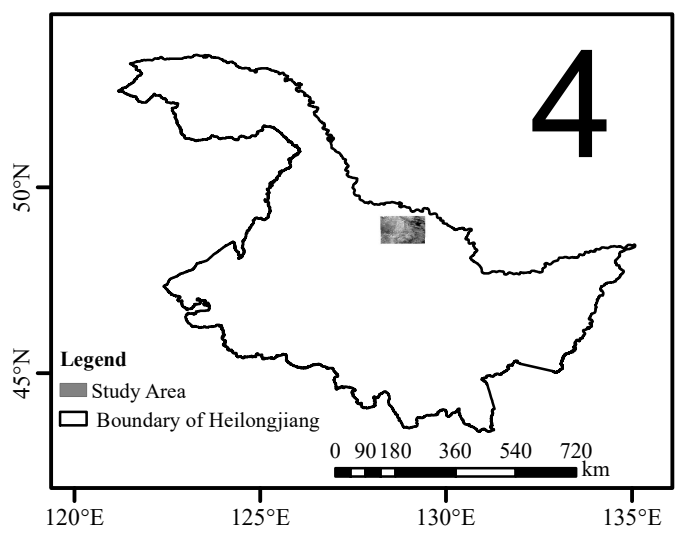

Figure 1. The schematic map of study area

Five kinds of Armillaria mellea are common in Northeast China, namely A. sinapina Brerube \& Dessur., A. galli-caMarxm \& Romagn., A. ostoyae (Romagn.) Herink, Chinese biological species $\mathrm{C}$ (CBSC) and Chinese biological species $\mathrm{F}$ (CBSF).Hazelnut mushroom can facultatively parasitize a variety of woody and herbal plants, mostly clumped at the base of old or dead trees, and can also parasitize living trees. It grows abundantly in shrubs and grasslands. Hazelnut mushroom is one of the most popular wild fungi in Northeast China because of its delicious taste and rich nutrition. It is rich in calcium, phosphorus, iron and other trace elements as well as protein, carotene, vitamin $\mathrm{C}$ and other nutrients, whose content is more than ten times that of ordinary vegetables. Hazelnut mushroom is classified as a kind of food in some developed countries. Hazelnut mushroom is smooth, delicious and nutritious. Hazelnut mushroom is umbrella-shaped, light earthy yellow, brown after aging. From July to September, Hazelnut mushroom grows on the base of coniferous and broad-leaved tree trunk, cutting roots, fallen trees and buried branches in the soil. It usually grows on hazelnut hills in shallow mountainous areas, so it is named Hazelnut mushroom(Figure 2).

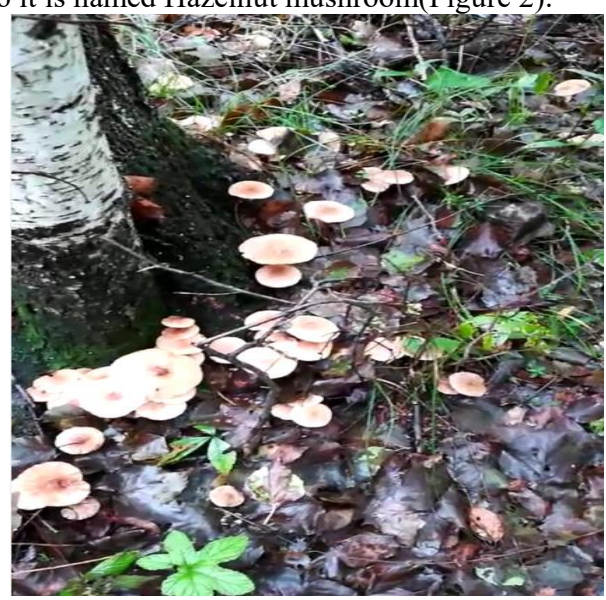

Figure 2. Growth picture of Armillariella mellea

\section{THE METHOD OF THE RESEARCH}

\subsection{The Method of Atmospheric Correction}

The principle of atmospheric correction method is to estimate the influence of atmosphere on the surface thermal radiation firstly, then subtract the influence from the total amount of thermal radiation observed by satellite sensors, so as to obtain the surface thermal radiation intensity, and then convert this thermal radiation intensity into the corresponding surface temperature.

The thermal infrared radiation brightness received by satellite sensor consists of three parts: the upward radiation brightness of the atmosphere, the real radiation brightness of the ground after passing through the atmosphere to reach the energy of the satellite sensor, and the energy reflected by the downward radiation of the atmosphere after reaching the ground. The expression of thermal infrared radiance received by satellite sensors can be written as (radiation transfer equation):

$$
L_{\lambda}=\left[\varepsilon B\left(T_{S}\right)+(1-\varepsilon) L \downarrow\right] \tau+L \uparrow
$$

where $\mathrm{L} \uparrow=$ the upward radiation brightness of the atmosphere atmosphere

L $\downarrow=$ the downward radiation brightness of the

$$
L_{\lambda}=\text { the thermal infrared radiance }
$$

$\mathrm{T}_{\mathrm{S}}=$ the real surface temperature

$\mathrm{B}\left(\mathrm{T}_{\mathrm{S}}\right)=$ the blackbody thermal radiation brightness $\tau=$ atmospheric transmittance in thermal infrared band object coordinates

The radiation brightness $\mathrm{B}(\mathrm{TS})$ of the blackbody with temperature $\mathrm{T}$ in the thermal infrared band is as follows:

$$
B\left(T_{S}\right)=\left[L_{\lambda}-L \uparrow-\tau(1-\varepsilon) L \downarrow\right] / \tau \varepsilon
$$

Ts can be obtained by the function of Planck's formula. 


$$
T_{S}=K_{2} / \ln \left(K_{1} / B\left(T_{S}\right)+1\right)
$$

For $\mathrm{TM}, \mathrm{K}_{1}=607.76 \mathrm{~W} /\left(\mathrm{m}^{2} * \mu \mathrm{m} * \mathrm{sr}\right), \mathrm{K}_{2}=1260.56 \mathrm{~K}$.

For $\mathrm{ETM}+, \mathrm{K}_{1}=666.09 \mathrm{~W} /\left(\mathrm{m}^{2} * \mu \mathrm{m} * \mathrm{sr}\right), \mathrm{K}_{2}=1283.71 \mathrm{~K}$.

For TIRS Band $10, \mathrm{~K}_{1}=774.89 \mathrm{~W} /\left(\mathrm{m}^{2} * \mu \mathrm{m} * \mathrm{sr}\right), \mathrm{K}_{2}=1321.08 \mathrm{~K}$.

Thus, two parameters are needed for this kind of algorithm: atmospheric profile parameters and surface emissivity. Atmospheric profile parameters can be obtained by inputting shading time and central longitude and latitude in the website provided by NASA (http://atmcorr.gsfc.nasa.gov/). It is suitable for data with only one thermal infrared band, such as Landsat $\mathrm{TM} / \mathrm{ETM}+/ \mathrm{TIRS}$ data. The main content is to use the BandMath tool to calculate formulas (2) and (3). The processing flow is shown in the figure 3 :

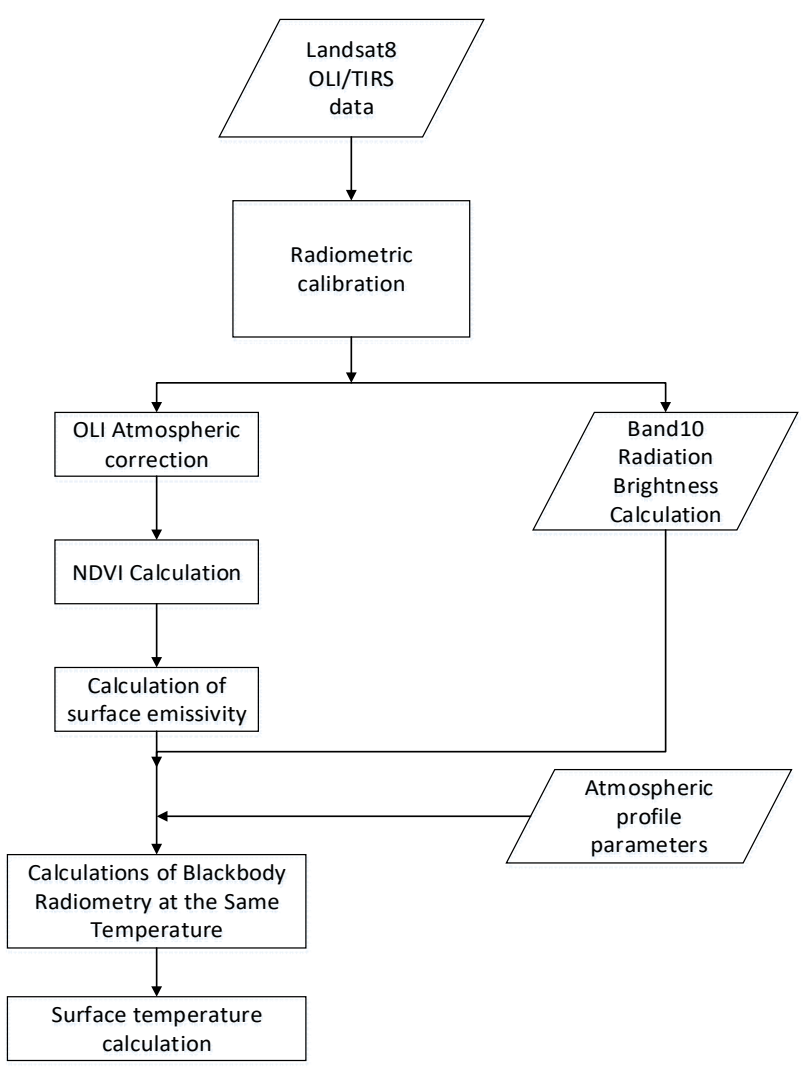

Figure 3. Flow chart of LST calculation

Finally, the surface temperature image is obtained(Figure 4).

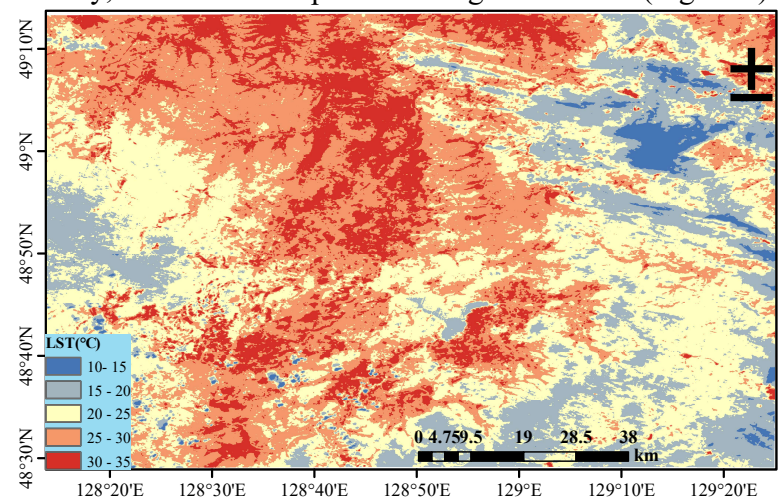

Figure 4. The schematic map of study area's LST

\subsection{The Method of Temperature Vegetation Dryness Index}

The Vegetation Drought Index Temperature Vegetation Dryness Index(TVDI) was retrieved from the surface soil moisture of vegetation coverage area using optical and thermal infrared remote sensing channel data. The TVDI, which is related to both normalized vegetation index (NDVI) and surface temperature (LST), can be used for drought monitoring, especially for monitoring the relative drought degree of the whole region in a given year, and for studying the spatial variation characteristics of drought degree.

Remote sensing radiation temperature will be affected by many factors such as vegetation coverage, evapotranspiration, surface thermal characteristics (thermal conductivity and heat capacity), net radiation, surface roughness and wind speed. There is a correlation between vegetation coverage and spectral vegetation index, and vegetation coverage determines that the sensor receives visible and thermal infrared information of soil background and vegetation canopy, thus affecting the radiation temperature obtained by remote sensing. There is no direct relationship between soil moisture status and surface temperature, but soil moisture is undoubtedly an important factor affecting vegetation canopy temperature. In this sense, canopy temperature under certain vegetation coverage can indirectly reflect the situation of soil water supply.

The formula of TVDI is:

$$
T V D I=\left(T_{S}-T_{\text {SMIN }}\right) /\left(T_{\text {SMAX }}-T_{\text {SMIN }}\right)
$$

The wet edge equation is

$$
T_{\text {SMIN }}=\left(a+b^{*} N D V I\right)
$$

The dry edge equation is

$$
T_{\text {SMIN }}=(c+d * N D V I)
$$

The range of TVDI is $[0,1]$.Thus, the linear relationship between the normalized vegetation cover index and the surface temperature was obtained based on the data of known images and the obtained normalized vegetation cover index(Figure 5).

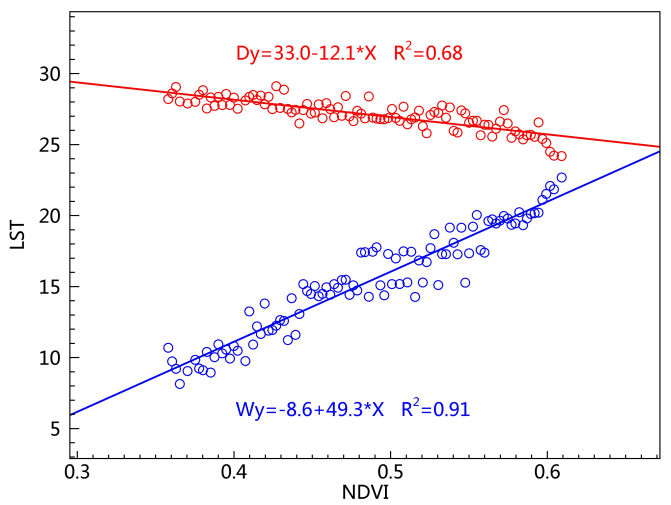

Figure 5. Linear Relation Diagrams of NDVI-LST

The higher the TVDI value, the lower the soil moisture, the smaller the TVDI, and the higher the soil moisture. TVDI can be obtained by using ENVI expansion technology with the obtained surface temperature and normalized vegetation index.Finally, the image of TVDI is obtained(Figure 6). 


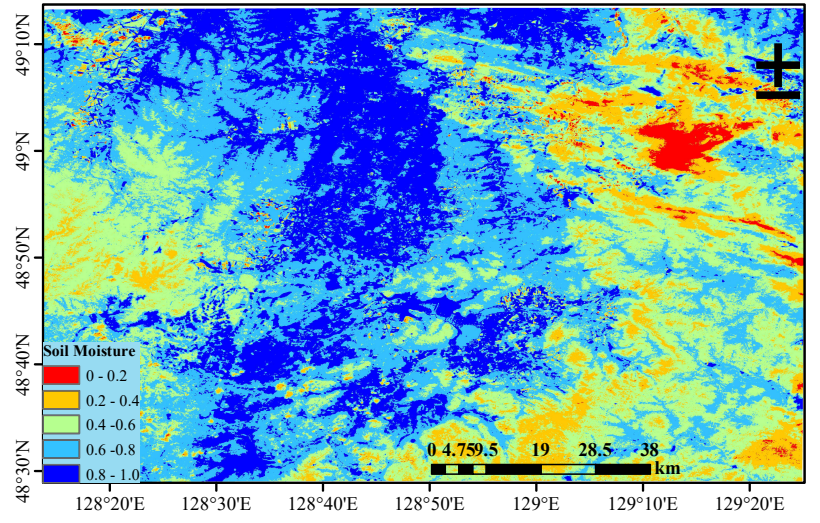

Figure 6 . The schematic map of study area's TVDI

\section{RESULTS AND ANALYSIS}

\subsection{The Growth of Armillariella Mellea in the Study Area}

Based on the analysis of surface temperature and soil moisture in the inverted study area, it was found that Hazelnut mushroom mainly grew in coniferous and broad-leaved forest with dense vegetation, moist soil and good light, especially in Hazelnut forest. In the study area, according to field survey and investigation, in late August 2014, hazelnut mushrooms grew in 82 of the 100 sampling sites surveyed. They mainly grew on the base of tree trunk, cutting, fallen trees and buried branches in the soil. They generally grew on hazelnut hills in shallow mountainous areas and grew well. Flake growth, in the sunny weather after the rain, hazelnut mushroom growth rapidly, if not harvested in time will rot in the forest, affecting the growth of vegetation.

\subsection{The Optimum Growth Temperature of Armillariella Mellea}

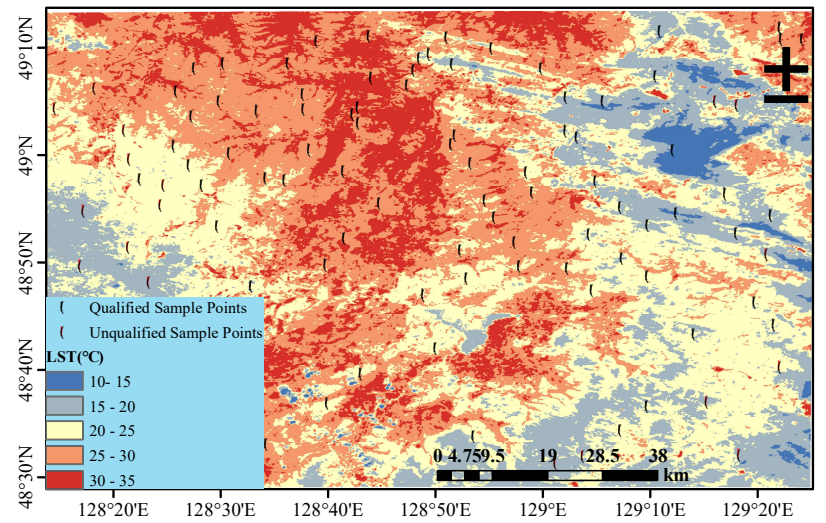

Figure 7. The Map with Samples in Study Area of LST

As shown in the figure, according to the surface temperature image obtained from the experiment, 8 places of Hazelnut mushroom grew in the temperature range of $15-20{ }^{\circ} \mathrm{C}$, accounting for $9.8 \%$ of the growing Hazelnut mushroom; 22 places of Hazelnut mushroom grew in the temperature range of $20-25{ }^{\circ} \mathrm{C}$, accounting for $26.8 \%$ of the growing Hazelnut mushroom; 36 places of Hazelnut mushroom grew in the temperature range of $20-25{ }^{\circ} \mathrm{C}$, accounting for $43.9 \%$ of the growing Hazelnut The mushroom grew in the temperature range of $20-25{ }^{\circ} \mathrm{C}$, accounting for $19.5 \%$ of the growth of Hazelnut mushroom. Hazelnut mushroom seldom grows at temperatures below 15 and its growth density decreases at $30-35{ }^{\circ} \mathrm{C}$. Hazelnut mushroom mainly grows at temperatures of $20-30{ }^{\circ} \mathrm{C}$. Most of them grow at temperatures of $25-30{ }^{\circ} \mathrm{C}$. With the increase of temperature, the growth density of Hazelnut mushroom increased gradually. The growth density of Hazelnut mushroom decreased when the temperature reached above 30 ${ }^{\circ} \mathrm{C}$. It can be seen that the growth of Hazelnut mushroom has certain requirements for temperature. Low and high temperatures are not conducive to the growth of Hazelnut mushroom. The optimum growth temperature of Hazelnut mushroom is in the range of $25-30{ }^{\circ} \mathrm{C}$

\subsection{The Optimum Growth humidity of Armillariella Mellea}

Soil moisture images obtained by inversion of soil moisture in the study area showed that there were 2 growth points of Hazelnut mushroom in the range of $0-0.2$ soil moisture, 20 growth points of Hazelnut mushroom in the range of 0.2-0.4 soil moisture, 35 growth points of Hazelnut mushroom in the range of 0.4-0.6 soil moisture, and 15 growth points in the range of $0.6-0.8$ soil moisture. There are 10 growth points of Hazelnut mushroom in the range of $0.8-1.0$ soil moisture. Hazelnut mushroom mainly grows in wet soil environment; in the range of soil moisture 0.2-0.6, the growth density of Hazelnut mushroom increases with the gradual decrease of soil moisture value; when soil moisture reaches below 0.2 , the growth density of Hazelnut mushroom decreases, indicating that when soil moisture reaches a certain peak, excessive water content is not conducive to Hazelnut mushroom. Growth. The optimum growth humidity of Hazelnut mushroom is between 0.2 and 0.6 (Figure 8)

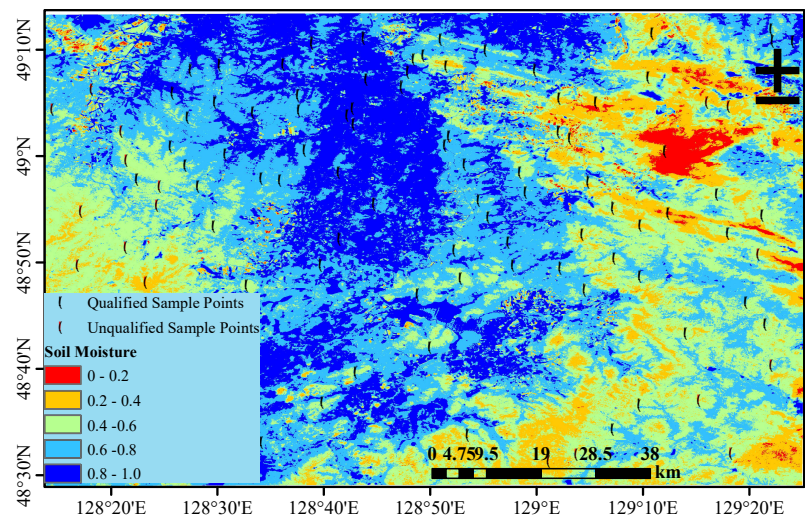

Figure 8. The Map with Samples in Study Area of TVDI

\section{CONCLUSION}

It is feasible to analyze the growth conditions of Hazelnut mushroom by retrieving surface temperature and soil vegetation index using remote sensing technology combined with field investigation. The accuracy of sampling survey reached $82 \%$. The optimum growth temperature and humidity of Hazelnut mushroom were $25-30{ }^{\circ} \mathrm{C}$ and $0.6-0.8$ respectively. Monitoring the growth environment of Hazelnut mushroom by remote sensing technology is of great significance. It can not only predict the growth of Hazelnut mushroom every year, but also provide a strong scientific basis for the artificial cultivation of Hazelnut mushroom, so that people can better and rationally use wild Hazelnut mushroom to realize the nutritional value of Hazelnut mushroom. 


\section{REFERENCES}

An X.L.,Wu J.J.,Zhou H.K.,2017,Applicability Analysis of Soil Relative Humidity in Agricultural Drought Monitoring in Northeast China.Geographical Research,36(5):837-849.

Gao W.,An R.,Wang Z.,2017.Drought Monitoring Index Based on Microwave Remote Sensing Technology and Its Application: A Case Study of Sanjiang Source Area.Study on Arid Areas,34(03):541-550.

Holzman M.E., Rivas R., Piccolo M.C.,2014. Estimating Soil Moisture and the Relationship with Crop Yield Using Surface Temperature and Vegetation Index. International Journal of Applied Earth Observation and Geoinformation,28:181-192.

Jimenez-Munoz J.C., Cristobal J., Sobrino J.A.,2009 Revision of the Single-Channel Algorithm for Land Surface Temperature Retrieval From Landsat Thermal-Infrared Data. IEEE Transactions on Geoscience and Remote Sensing, 47(1):339349.

Qin Z.H.,Minghua Z. , Karnieli A., 2001.Single-window Algorithm for Calculating Surface Temperature From Landsat TM6 data.Journal of Geography, 68(4).

Rozenstein O., Qin Z., Derimian Y., 2014, Derivation of Land Surface Temperature for Landsat-8 TIRS Using a Split Window Algorithm. Sensors, 14(4):5768-5780.

Wang X.,Liu Q.M.,Qu Z.Y.,2017.Microwave Radar Retrieval and Verification of Saline Soil Moisture.Journal of Agricultural Engineering,2017(11):116-122.

Wu B.F.,Zhang F.,Liu C.L.,2004.Integrated Remote Sensing Monitoring Method for Crop Growth.Journal of Remote Sensing,2004(06):498-514.

Yan F.,Qin Z.H.,Li M.S.,2006.Advances in Soil Moisture Retrieval by Remote Sensing in Agricultural Drought Monitoring.Journal of Natural Disasters, 15(06):114-121.
Yang S.Y.,Lin Y.C.,Yi H.,2007.Development and Application of Armillaria Mellea, - a Rare Edible and Edicinal Fungus.Journal of Biology,2007(03):52-54

Yu J.,Yang G.F.,Wang Y.,2011.Retrieval of Soil Moisture in Fuxin Area Based on MODIS Data.Remote Sensing Technology and Application, 26(4):413-419.

Zheng H.C.,Cui W.,Li S.L.,2014.Investigation on the Growth Environment of Wild Hazelnut Mushroom in Hailin Area of Heilongiiang Province.Anhui Agricultural Science,42(36).

Zhou L., Zhang J., Wu J.,2012. Comparison of Remotely Sensed and Meteorological Data-derived Drought Indices in Mid-eastern China. International Journal of Remote Sensing, 33(6):1755-1779.

Zhu S.Y.,Zhang G.X.,Yin Q,2006.Research Status and Development Trend of Land Surface Temperature Retrieval by Thermal Infrared Remote Sensing.Remote Sensing Technology and Application, 21(5):420-425.

Zhu Z.R.,Cheng P.G.,Gui X.,2016.A Summary of Algorithms for Land Surface Temperature Inversion.Surveying and Mapping and Spatial Geographic Information,39(5):70-75. 